\title{
Magnetomechanical properties of a high-temperature Ni-Mn-Ga magnetic shape memory actuator material
}

\author{
E. Pagounis, ${ }^{\mathrm{a}, *}$ A. Laptev, ${ }^{\mathrm{b}}$ J. Jungwirth, ${ }^{\mathrm{b}}$ M. Laufenberg ${ }^{\mathrm{a}}$ and M. Fonin ${ }^{\mathrm{b}}$ \\ ${ }^{\mathrm{a}}$ ETO MAGNETIC GmbH, Hardtring 8, 78333 Stockach, Germany \\ ${ }^{\mathrm{b}}$ Fachbereich Physik, Universität Konstanz, 78457 Konstanz, Germany
}

\begin{abstract}
A Ni-Mn-Ga single crystal with a phase transformation temperature above $350 \mathrm{~K}$ is investigated. The material demonstrates a magnetic field-induced strain (MFIS) of $7 \%$ at room temperature and of $4.2 \%$ at $350 \mathrm{~K}$. The temperature dependence of the magnetic anisotropy and of the saturation magnetization has been studied in detail. At $350 \mathrm{~K}$ a maximum magnetic stress of 1.6 $\mathrm{MPa}$ and a twinning stress of 0.6 MPa were measured, explaining the MFIS obtained at elevated temperatures.
\end{abstract}

Keywords: Ferromagnetic shape memory; Magnetic properties; Twinning; High-temperature strain; Actuator

Magnetic shape memory (MSM) actuation has been studied as a novel approach to generate motion and force. In MSM materials the reorientation and motion of martensite twin boundaries at moderate magnetic fields of $<1 \mathrm{~T}$ can, depending on the crystal structure, produce shape changes of $>6 \%$, accompanied by a magnetically induced stress of $>2 \mathrm{MPa}[1]$. Using these materials energy-efficient actuators can be designed with extremely short response times and with a work output exceeding that of other actuator materials [1-3]. Typically, MSM materials are produced in single-crystalline form; however, textured polycrystals, composites, foams and thin films have also been investigated [4-6]. Today, the most widely explored MSM materials are Heuslertype off-stoichiometric $\mathrm{Ni}_{2} \mathrm{MnGa}$ alloys. Here, the martensitic structure plays a key role in governing the mechanical and magnetic properties. Three martensitic phases have been described in these alloys, namely the $10 \mathrm{M}$ and $14 \mathrm{M}$ modulated ones and the non-modulated tetragonal martensite $[7,8]$. Which of these structures predominates depends on the chemical composition and the prior thermal history of the alloy. For the time being, the $10 \mathrm{M}$ structure has proven the most suitable

\footnotetext{
* Corresponding author. Tel.: +49 7771809468; e-mail: e.pagounis@ etogroup.com
}

one for practical applications due to its low twinning stress and high work output $[9,10]$.

In recent years considerable improvements have been achieved in the mechanical stability, work output and production of these materials [11-13]. Furthermore, actuators, sensors and power generators have been designed based on MSM materials [14,15]. High-temperature $\mathrm{Ni}-\mathrm{Mn}-\mathrm{Ga}$ alloys, with phase transformation temperatures of up to $500 \mathrm{~K}$, have been studied too, because of their promising superelastic properties and shape memory effect $[16,17]$. These materials, typically with high Ni content and a phase transformation temperature considerably higher than the Curie point, are, however, magnetically inactive. The development of high-temperature MSM alloys demonstrating magnetic field-induced strain (MFIS) both at room and at high temperature has been the research target of several studies over the past two decades. However, little progress has been achieved. In MSM materials the maximum operating temperature is limited by the martensite-to-austenite transformation temperature and by magnetomechanical properties, including the magnetocrystalline anisotropy energy and the twinning stress. Large magnetocrystalline anisotropy is a key requirement for MFIS because the driving force for twin reorientation is the different total energy of martensite twin variants in a magnetic field. In addition, a low twinning stress is necessary for the twin boundary motion to be induced by the magnetic field. Until 
recently the maximum operating temperature of $\mathrm{Ni}-\mathrm{Mn}$ Ga MSM materials was well below $327 \mathrm{~K}$ [18], which limits their application potential. Two of the present authors have recently discovered a $\mathrm{Ni}-\mathrm{Mn}-\mathrm{Ga}$ material demonstrating MFIS at elevated temperatures $>350 \mathrm{~K}$ and have analyzed its crystal structure [19]. In the present paper, the magnetomechanical properties of a hightemperature MSM material are investigated in detail. It is further demonstrated that the material gives an effective work output suitable for actuator applications.

Single-crystalline samples with the composition of Ni50.8-Mn28.3-Ga20.9 ( \pm 0.5 at. $\%)$ were produced using Bridgman-type crystal growth at ETO MAGNETIC $\mathrm{GmbH}$. The crystals were homogenized and ordered at temperatures close to $1273 \mathrm{~K}$. They were then oriented and cut by spark erosion to produce rectangular samples with the $\{100\}$ planes of the high-temperature cubic phase normal to the sample surface. The samples were trained to obtain a single-variant state for magnetomechanical measurements.

The sample used for SQUID magnetization measurements was prism-shaped with dimensions of about $2 \times 2 \times 3 \mathrm{~mm}^{3}$. Temperature-dependent measurements of the magnetization were performed with a Quantum Design MPMS XL5 SQUID magnetometer. Two different magnetic fields ( $10 \mathrm{mT}$ and $2 \mathrm{~T})$ were applied in order to deduce the phase transformation temperatures and the saturation magnetization. Before starting the measurements the sample was compressed along the [0 011$]$ direction to achieve the single-variant state. The field warming (FW) was then carried out first followed by the field cooling (FC) measurement. The heating/cooling rate was $0.2 \mathrm{~K} \mathrm{~min}^{-1}$.

Magnetization curves for determining the anisotropy constants were measured at three different temperatures, namely 300,330 and $348 \mathrm{~K}$. The maximum temperature was selected in order for the sample to remain in the martensitic state. To ensure that the sample is in a single-variant state, which is required for measuring the anisotropy constants, the sample was compressed prior testing along the $[001]$ direction. During the measurements, carried out in all three $\langle 100\rangle$ crystallographic directions, the sample was mechanically constrained to avoid MFIS. The magnetization curves were corrected for the demagnetization field taking into account the shape of the sample. The magnetic anisotropy constants were then calculated from the area between the curves.

Magnetomechanical measurements to determine the MFIS, the twinning stress, the magnetic stress, etc., were carried out using a water-cooled electromagnet (GMW, San Carlos, CA) capable of generating fields of up to $2 \mathrm{~T}$ within an air gap of up to $40 \mathrm{~mm}$. The samples used for these measurements had the dimensions of about $2 \times 3 \times 15 \mathrm{~mm}^{3}$. Here the magnetic field was applied normal to the $3 \times 15 \mathrm{~mm}^{2}$ surface of the sample, i.e. along the $\left[\begin{array}{lll}1 & 0 & 0\end{array}\right]$ crystallographic direction. The elongation was then obtained perpendicular to the applied field and along the sample's long axis. Compression tests were performed with a Zwick-Roell testing apparatus and a load cell of $2.5 \mathrm{kN}$. For the high-temperature measurements the samples were introduced in a copper holder, which was heated and cooled using a Julabo F 32 thermostat. The sample temperature was monitored with an accuracy of about $\pm 2 \mathrm{~K}$ by a thermocouple attached at the bottom of the sample. The magnetic field was measured with a Magnet-Physik FH 55 gaussmeter equipped with a Hall sensor, which was placed at a predefined position on the sample surface. In the strain-field curves the sample's elongation in the magnetic field was measured with a laser triangulation sensor (Micro-Epsilon), operating at a sampling rate of $37 \mathrm{kHz}$. In the stress-strain curve the stress and strain output was monitored using the relevant sensors of the Zwick set-up.

The temperature behavior of the magnetization was studied over the temperature range from 300 to $400 \mathrm{~K}$ and in magnetic fields of $10 \mathrm{mT}$ and $2 \mathrm{~T}$ (Fig. 1). The phase transformation temperatures were derived from the low-field measurement. The martensite-to-austenite transformation starts at $A_{s}=351 \mathrm{~K}$ and ends at $A_{f}=353 \mathrm{~K}$. The austenite-to-martensite transformation starts at $M_{s}=344 \mathrm{~K}$ and ends at $M_{f}=330 \mathrm{~K}$. A narrow thermal hysteresis of $\sim 9 \mathrm{~K}$ is thus obtained. This indicates that the martensitic transformation is thermoelastic and that the driving force for the transformation is small $[18,20]$. A further detailed study of the transformation behavior of the high-temperature alloy is planned by the authors. The Curie temperature is at $T_{C}=373 \mathrm{~K}$, which, despite the increased phase transformation temperatures in the present alloy, is similar to results on samples with transformation temperatures slightly above room temperature [21]. After cooling from the austenite the material is in the multivariant state. This explains the higher magnetization of martensite in the FC curve as compared to that during FW.

The magnetization curve obtained in an external field of $2 \mathrm{~T}$ (Fig. 1), which is sufficient to saturate the sample (see Fig. 2), reveals the temperature behavior of the saturation magnetization $M_{S}$. A similar curve at fields above saturation has been reported in a $\mathrm{Ni}-\mathrm{Mn}-\mathrm{Ga}$ alloy with a martensitic transition at $306 \mathrm{~K}$ [22]. Since the saturation magnetization of martensite is higher than that of austenite [18], the phase transformation temperatures can be detected on the curves. In an external field of $2 \mathrm{~T}$ the austenite transformation temperature $A_{s}$ in the present alloy is shifted by $\sim 1 \mathrm{~K}$ to higher temperatures. This can be attributed to the stabilization of martensite when a sufficiently high magnetic field is applied [23]. The value of the saturation magnetization

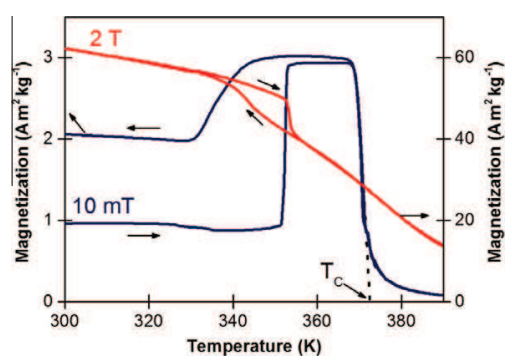

Figure 1. Temperature dependence of the magnetization measured in an external magnetic field of $10 \mathrm{mT}$ (left axis) and of $2 \mathrm{~T}$ (right axis). In both measurements, at $10 \mathrm{mT}$ and at $2 \mathrm{~T}$, the magnetic field was applied along the [010] direction of the single-variant sample. The magnetization measured in an external field of $2 \mathrm{~T}$ corresponds to the saturation magnetization of the sample. The Curie temperature $T_{C}$ of the $\mathrm{Ni}-\mathrm{Mn}-\mathrm{Ga}$ alloy is also shown. 


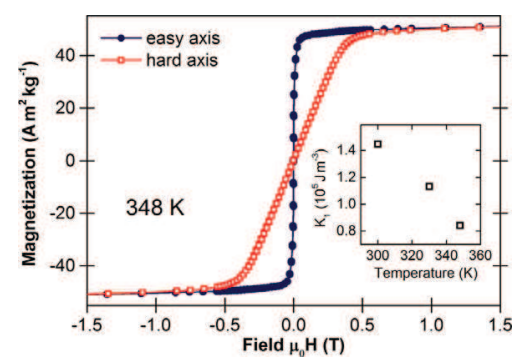

Figure 2. Magnetization as a function of applied magnetic field measured along the easy [001] and the hard [100] directions of the single-variant sample at $348 \mathrm{~K}$. The inset shows the obtained temperature dependence of the anisotropy constant $K_{1}$.

decreases from $M_{S}=61.2 \mathrm{~A} \mathrm{~m}^{2} \mathrm{~kg}^{-1}$ at $300 \mathrm{~K}$ to $M_{S}=51.2 \mathrm{~A} \mathrm{~m}^{2} \mathrm{~kg}^{-1}$ at $348 \mathrm{~K}$.

Magnetization curves as a function of the applied magnetic field were measured above room temperature, in the martensitic state of the sample, and in all three $\langle 100\rangle$ directions. As an example the magnetization curves for the highest temperature ( $348 \mathrm{~K}$, i.e. close to $A_{S}$ ) are shown in Figure 2. The curves obtained for the $\left[\begin{array}{lll}1 & 0 & 0\end{array}\right]$ and $\left[\begin{array}{lll}0 & 1 & 0\end{array}\right]$ crystallographic directions coincide for all studied temperatures. The curves measured in these directions show a linear and slow approach to saturation. The magnetization curve measured for the third crystallographic [0 0101$]$ direction is square-like with a fast approach to saturation. This magnetization behavior indicates that at the studied temperatures there are two equivalent axes of hard magnetization and one easy magnetization axis. This type of curve has been reported in 10M modulated martensites $[18,21]$. The calculated anisotropy constant $K_{1}$ was found to be $1.45 \times 10^{5} \mathrm{~J} \mathrm{~m}^{-3}$ at $300 \mathrm{~K}$. This value is slightly lower compared to that measured in Ni-Mn-Ga alloys with a 10M martensitic structure, which have transformation temperatures of $\sim 317 \mathrm{~K}[24,25]$. With increasing temperature the anisotropy constant $K_{1}$ decreases to $0.84 \times 10^{5} \mathrm{~J} \mathrm{~m}^{-3}$ at $348 \mathrm{~K}$ (Fig. 2 inset), in agreement with previous studies revealing a rapid decrease of magnetic anisotropy when approaching the Curie temperature $[25,26]$. Similar to the measurements carried out at 300 and $330 \mathrm{~K}$, and also at $348 \mathrm{~K}$, the magnetization curve in the hard direction (Fig. 2) shows an excellent linearity nearly to saturation, and thus the martensitic structure is confirmed.

In Figure 3, the strain as a function of the applied field at room and at high temperature is presented, demonstrating a MFIS of $4.2 \%$ at $350 \mathrm{~K}$. This value is close to that measured in Ref. [19] for a similar

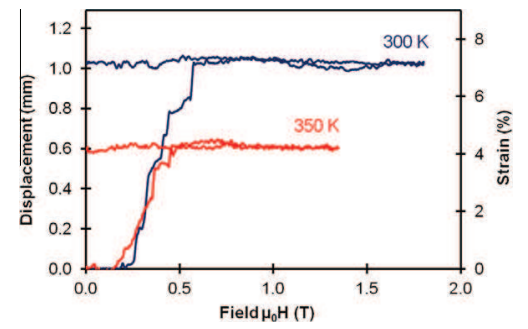

Figure 3. Strain as a function of applied magnetic field for the $\mathrm{Ni}-\mathrm{Mn}-\mathrm{Ga}$ MSM material at room and at high temperature. The field is applied along the [100] direction of the sample and the strain is measured perpendicular to the field. high-temperature MSM alloy. The magnetic field needed for maximum strain at both temperatures is below $0.6 \mathrm{~T}$ and within the range typically achieved in electromagnetic actuators. The slightly lower activation field in the high-temperature measurement can be attributed to a reduced twinning stress at elevated temperatures as well as to the increased thermal activation of twin-boundary motion $[18,27]$.

In Figure 4, the stress-strain curve over a complete actuation cycle at $350 \mathrm{~K}$ is presented. The material initially elongates in the presence of the magnetic field and the stress output is monitored. When the elongation reaches its maximum value at 3\% the field is switched off and the sample returns to its original shape by mechanical compression with the Zwick set-up. The stress-strain curve gives valuable information about the twinning stress $\sigma_{t w}$, the magnetic stress $\sigma_{M}$, the work output (force $\times$ displacement), and the actuation efficiency of the MSM material. The lower MFIS measured in Figure 4 compared to that in Figure 3 is attributed to the external stresses applied in the stress-strain curve. The twinning stress at $350 \mathrm{~K}$, deduced from the stress plateau at half the maximum strain after the magnetic field has been removed [18], is $\sigma_{t w}=0.6 \mathrm{MPa}$ (Fig. 4). It is by far the lowest twinning stress reported at elevated temperatures, contributing to a great extent to the obtained MFIS. The maximum available magnetic stress measured $\sigma_{M}^{\max }=1.6 \mathrm{MPa}$. This is considerably higher than the twinning stress, i.e. the condition $\sigma_{M}^{\max }>\sigma_{t w}$ for giant MFIS due to twin boundary motion is fulfilled. The efficiency of the sample during the actuation cycle is deduced from the ratio of the effective magnetic field induced work output, expressed by the area cross-section between the two curves in Figure 4, to the total work output. The effective work output of the sample measured $1 \mathrm{~N} \mathrm{~mm}$, while the total work output was $2.2 \mathrm{~N} \mathrm{~mm}$. Accordingly, for the alloy studied in the present work the actuation efficiency at $350 \mathrm{~K}$ is $45 \%$.

The positive value of the effective work output and the large efficiency at high temperature demonstrate that the present material is suitable for actuator applications. In the literature the strain-field curve is frequently used to demonstrate MFIS at zero pre-stress. This curve, however, does not provide sufficient information about the actuation properties of the MSM material. Even

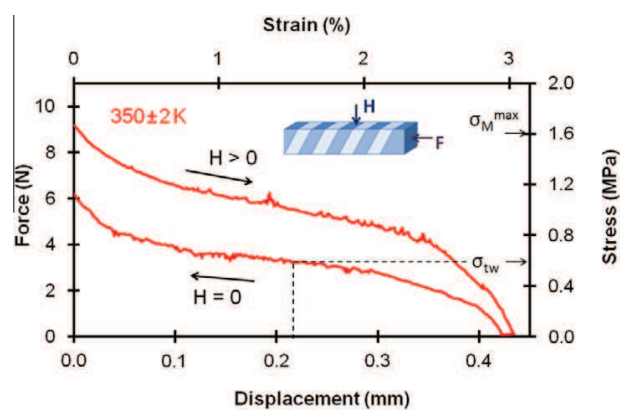

Figure 4. Stress-strain curve of the MSM sample during an actuation cycle at $350 \mathrm{~K}$. The material is elongated by a magnetic field of $\sim 1 \mathrm{~T}$ applied along the [100] direction and returns to its initial state, after the field has been switched off, by mechanical compression. The maximum magnetic stress $\sigma_{M}^{\max }$ and the twinning stress $\sigma_{t w}$ are also indicated. The twinning stress is measured at half the maximum strain. 
though MFIS at zero pre-stress is demonstrated, the MSM material might not be suitable for actuator applications because the mechanical stress needed to return the sample to its initial shape can be larger than the magnetic stress produced by the sample. Accordingly, the stress-strain curve such as in Figure 4 is more useful to directly demonstrate the actuation potential than the more widely used strain-field curve as in Figure 3.

A well-established MSM model has been proposed [28], which correlates the magnetic anisotropy constant $K_{1}$ with the maximum magnetic stress $\sigma_{M}^{\max }$ and the maximum theoretical strain due to lattice distortion $\varepsilon_{0}$ as follows: $\sigma_{M}^{\max }=K_{1} / \varepsilon_{0}$. The anisotropy measurements in the present study, as well as the XRD investigation of a similar alloy in Ref. [19], indicate that the material has a $10 \mathrm{M}$ martensitic structure at $\sim 350 \mathrm{~K}$, with $\varepsilon_{0}=6 \%$. Based on the MSM model, the maximum available magnetic stress at this temperature is calculated to be $\sigma_{M}^{\max }=0.84 \times 10^{5} \mathrm{~J} \mathrm{~m}^{-3} / 0.06=1.4 \mathrm{MPa}$. This is close to the experimentally measured value of $1.6 \mathrm{MPa}$ (Fig. 4). The small difference of $0.2 \mathrm{MPa}$ can be attributed to the effect of external stresses, e.g. friction and stresses arising from the sample fixation during testing, which can slightly modify the measurement results. Similar observations have been reported elsewhere [18].

In conclusion, a high-temperature $\mathrm{Ni}-\mathrm{Mn}-\mathrm{Ga}$ alloy is presented that demonstrates giant MFIS over the temperature range up to $350 \mathrm{~K}$. Magnetomechanical measurements have been carried out to characterize the material's actuation properties. The temperature dependence of the magnetocrystalline anisotropy and of the saturation magnetization has been investigated in detail. A low twinning stress of $0.6 \mathrm{MPa}$ and a large magnetic stress of $1.6 \mathrm{MPa}$ have been measured at $350 \mathrm{~K}$. These values justify the obtained high-temperature MFIS of up to $4.2 \%$, which is attributed to the martensite twin boundary motion. The material studied here is suitable for actuator applications, and demonstrates large work output and efficiency during an actuation cycle at elevated temperatures.

The authors would like to thank M. Helmer and M. Maier of ETO MAGNETIC GmbH for sample preparation and testing.

[1] E. Pagounis, Mater. Sci. Eng., R 56 (2007) 33.

[2] Y. Ganor, D. Shilo, T.W. Shield, R.D. James, Appl. Phys. Lett. 93 (2008) 122509.
[3] E. Pagounis, H. Schmidt, in Proc. Actuator 2012, Bremen, 2012, p. 317.

[4] D.C. Dunand, P. Müllner, Adv. Mater. 23 (2011) 216.

[5] P. Leicht, A. Laptev, M. Fonin, Y. Luo, K. Samwer, New J. Phys. 13 (2011) 033021.

[6] U. Gaitzsch, J. Romberg, M. Pötschke, S. Roth, P. Müllner, Scr. Mater. 65 (2011) 679.

[7] A.A. Likhachev, A. Sozinov, K. Ullakko, Mater. Sci. Eng., A 378 (2004) 513.

[8] N. Lanska, O. Söderberg, A. Sozinov, Y. Ge, K. Ullakko, V.K. Lindroos, J. Appl. Phys. 95 (2004) 8074.

[9] I. Suorsa, E. Pagounis, K. Ullakko, Sensor Actuat. A 121 (2005) 136.

[10] A. Sozinov, N. Lanska, A. Soroka, L. Straka, Appl. Phys. Lett. 99 (2011) 124103.

[11] F. Xiong, Y. Liu, E. Pagounis, J. Alloys Compd. 415 (2006) 188.

[12] E. Pagounis, M. Laufenberg, Patent Application EP 2339595 (14.12.2009).

[13] I. Aaltio, O. Söderberg, Y. Ge, S.P. Hannula, Mater. Sci. Forum 684 (2011) 201.

[14] I. Suorsa, J. Tellinen, I. Aaltio, E. Pagounis, K. Ullakko, in Proc. Actuator 2004, Bremen, 2004, p. 573.

[15] J. Stephan, E. Pagounis, M. Laufenberg, O. Paul, P. Ruther, IEEE Sensors J. 11 (2011) 2683.

[16] Y. Ma, C. Jiang, Y. Li, H. Xu, C. Wang, X. Liu, Acta Mater. 55 (2007) 1533.

[17] E. Cesari, J. Font, J. Muntasell, P. Ochin, J. Pons, R. Santamarta, Scr. Mater. 58 (2008) 259.

[18] L. Straka, Ph.D. thesis, Helsinki University of Technology, Espoo, 2007.

[19] E. Pagounis, R. Chulist, M.J. Szczerba, M. Laufenberg, Scr. Mater. 83 (2014) 29.

[20] M. Richard, J. Feuchtwanger, D. Schlagel, T. Lograsso, S.M. Allen, R.C. O'Handley, Scr. Mater. 54 (2006) 1797.

[21] L. Straka, O. Heczko, J. Appl. Phys. 93 (2003) 8636.

[22] F.Q. Zhu, F.Y. Yang, C.L. Chien, L. Ritchie, G. Xiao, G.H. Wu, J. Magn. Magn. Mater. 288 (2005) 79.

[23] V.A. Chernenko, V.A. Lvov, T. Kanomata, T. Kakeshita, K. Koyama, S. Besseghini, Mater. Trans. 47 (2006) 635.

[24] S.J. Murray, M. Marioni, S.M. Allen, R.C. ÓHandley, T.A. Lograsso, Appl. Phys. Lett. 77 (2000) 886.

[25] O. Heczko, L. Straka, N. Lanska, K. Ullakko, J. Enkovaara, J. Appl. Phys. 91 (2002) 8228.

[26] F. Albertini, L. Pareti, A. Paoluzi, L. Morellon, P.A. Algarabel, M.R. Ibarra, L. Righi, Appl. Phys. Lett. 81 (2002) 4032.

[27] R.C. O'Handley, D.I. Paul, S.M. Allen, M. Richard, J. Feuchtwanger, B. Peterson, R. Techapiesancharoenkij, M. Barandiarán, P. Lázpita, Mater. Sci. Eng., A 438-440 (2006) 445.

[28] A. Likhachev, K. Ullakko, Phys. Lett. A 275 (2000) 142. 